Rapid Communications

\title{
Anomalous Fluorescent Responses of 9-Fluorenecarboxamide Derivatives Possessing Benzo-15-crown-5
}

\author{
Hiroya Hama, * Tatsuya Morozumi,** and Hiroshi NAKaMura** \\ *Division of Environmental Material Science, Graduate School of Environmental Science, \\ Hokkaido University, Sapporo 060-0810, Japan \\ **Section of Materials Science, Research Faculty of Environmental Earth Science, \\ Hokkaido University, Sapporo 060-0810, Japan
}

\begin{abstract}
A new fluorescent ionophore for alkaline earth metal ions, 4'-(9-fluorenecarboxamido)benzo-15-crown-5 (3), was synthesized; its photochemical behavior was investigated using UV, fluorescence, ${ }^{1} \mathrm{H}$ NMR, and ${ }^{13} \mathrm{C}$ NMR spectrometry. In the absence of a metal ion, the fluorene moiety showed weak fluorescence emission (fluorescence "Off" state). However, complexation with metal ions greatly enhanced the fluorescence intensity (fluorescence "On" state).
\end{abstract}

(Received September 18, 2007; Accepted October 17, 2007; Published November 10, 2007)

The importance of electron-transfer reactions has attracted many researchers to investigate the fundamental steps of the processes. Especially, many studies have been devoted to photo-induced electron transfer (PET) in electron donoracceptors linked by a covalent bond system. ${ }^{1}$ In that context, we have reported photochemical behavior of a series of donoracceptor systems. Recently, we reported that some aromatic amide derivatives that have fluorescent active groups in the molecules showed quite weak emission. The presence of $N$ phenyl-amide groups caused the formation of charge separation at the excited state through electron transfer between fluorescence-active groups and a benzene ring via the amide bond. Using results of a previous study, new fluorescent ionophores based on an ethyleneoxy chain containing $N$-phenyl9-anthracenecarboxamide ${ }^{2}$ and $\mathrm{N}$-phenyl-1-pyrenylcarboxamide ${ }^{3}$ were synthesized and characterized. These fluoroionophores showed weak emission corresponding to their monomers in the absence of metal ions, although their alkaline earth metal ion complexes indicated strong emissions. Their photochemical action of complexation suggested that the most likely relaxation pathway was twisted intramolecular charge transfer (TICT). To extend these investigations, we synthesized novel chemosensors based on benzo-crown ether ${ }^{4}$ and linear polyether ${ }^{5}$ possessing $N$-phenyl-1-naphthalenelacetamide. These sensors indicated that the binding of alkaline earth metal ions is signaled by a switching "on" of the naphthalene fluorescence. Fluorescence spectroscopic data suggest that their quenching process was intermolecular charge transfer involving a TICT character. These results might be construed to mean that TICT has occurred not only through the amide bond but also over the $\mathrm{CH}_{2}$ group. The concept of TICT usually emphasizes the torsion of a single bond, although the CT action of these cases was obtained over the two bonds (amide and $\mathrm{CH}_{2}$ group). The charge-transfer behavior of $\mathrm{N}$-phenyl-1-naphthaleneacetamide was unique.

We used 9-fluorenecarboxamide as a comparison to confirm that this CT action is peculiar to naphthalene. Fluorene has a similar UV absorption band to that of naphthalene, in addition to a unique bridge carbon out of the conjugation constructed by the biphenyl moiety. A similar UV absorption spectrum would probably use the same excited-state. The nine-positioned carbon should provide evidence for considering of the effects of the donor-acceptor distance. Fluorene is also a promising material for organic light-emitting devices because of its good chemical stability and high photoluminescence quantum efficiency, both in solution and in the solid state. ${ }^{6}$ In addition, in the biochemical field, it has been used as "molecular beacons" for detecting nucleic acid hybridization. ${ }^{7,8}$ Despite their advantages, applications for analytical use and signaling purposes using fluorene are rather limited. In this communication, using fluorescence, UV, and NMR spectrometries, we describe the photochemical behaviors
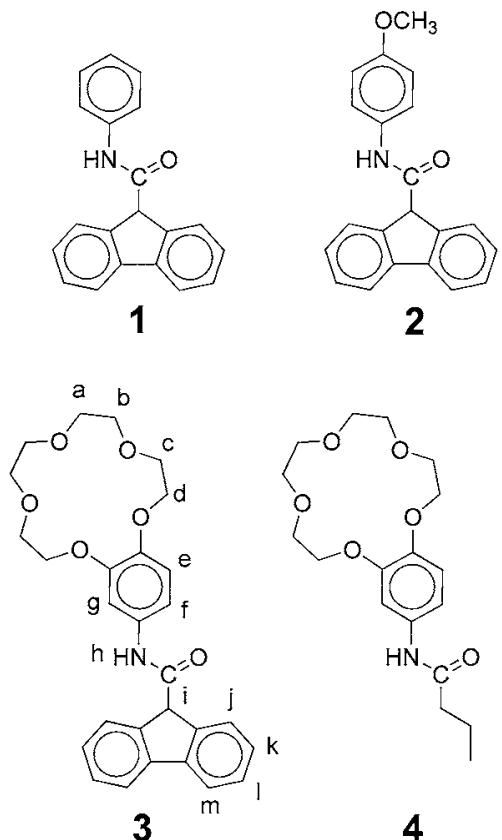

Fig. 1 Structures of $\mathbf{1}, \mathbf{2}, \mathbf{3}$, and $\mathbf{4}$. 


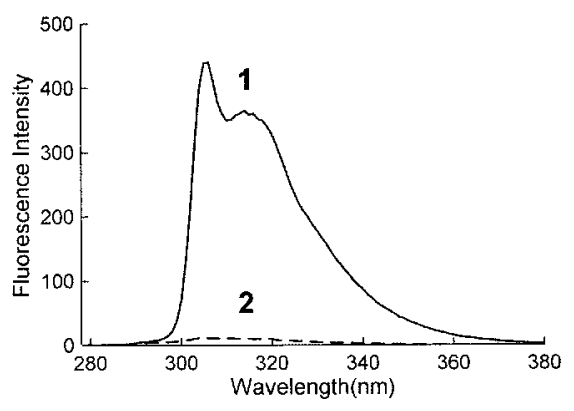

Fig. 2 Fluorescence spectra of $\mathbf{1}$ (solid line) and $\mathbf{2}$ (chain line) in acetonitrile at $25^{\circ} \mathrm{C}$. Excitation wavelength: $268 \mathrm{~nm}$. [1] $=[2]=5 \times$ $10^{-6} \mathrm{M}$. The respective slits for the excitation and emission monochromators were 3.0 and $1.5 \mathrm{~nm}$.

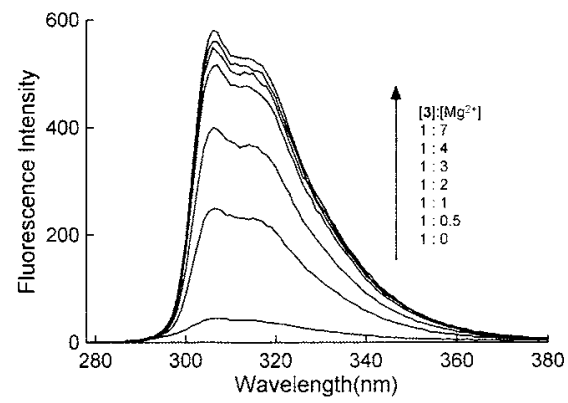

Fig. 3 Fluorescence spectra of $\mathbf{3}$ and its $\mathrm{Mg}^{2+}$ complex in acetonitrile at $25^{\circ} \mathrm{C}$. Excitation wavelength: $268 \mathrm{~nm}$. [3] $=5 \times 10^{-6}$ M. The respective slits for the excitation and emission monochromators were 3.0 and $5.0 \mathrm{~nm}$.

of a new fluorescent benzo-crown ether possessing 9-fluorenecarboxamide 3 (Fig. 1) upon complexing with metal ions.

\section{Experimental}

Ionophore $\mathbf{3}$ was synthesized in the usual manner from 1-fluoreneacetic acid with the corresponding amine using DCC as a coupling agent in the presence of HOBT (57.5\% yield). ${ }^{9}$ This structure and purity were confirmed using ${ }^{1} \mathrm{H}$ and ${ }^{13} \mathrm{C}$ NMR spectra and elemental analysis. Fluorescence spectra were measured using a spectrometer (RF-5300PC; Shimadzu Corp.) at $25^{\circ} \mathrm{C}$. The concentration of the fluorescent reagent was $5 \times 10^{-6} \mathrm{~mol} / \mathrm{dm}^{3}$ in purified acetonitrile. Alkaline earth metal cations were added to the fluorescent reagent solution as perchlorate salts.

\section{Results and Discussion}

The fluorescence properties were checked using model compounds to aid in the interpretation concerning the photochemical action of 3 . Figure 2 indicates fluorescence spectra of $N$-phenyl-9-fluorenecarboxamide (1) and $N$-(4methoxy-phenyl)-9-fluorenecarboxamide (2) in acetonitrile solution. Despite their similar structures, fluorescent emissions from 2 were quite small. This result indicates that the electron donation property of the methoxy group played an extremely important role in fluorescent quenching.
Table 1 Complex formation constants $(K)^{\mathrm{a}}$ and fluorescent response $\left(I_{\max } / I_{0}\right)$ of $\mathbf{3}$ for various metal ions in acetonitrile at $25^{\circ} \mathrm{C}$

\begin{tabular}{cccc}
\hline \multicolumn{4}{c}{$\log K\left(I_{\max } / I_{0}\right)$} \\
\hline $\mathrm{Mg}^{2+}$ & $\mathrm{Ca}^{2+}$ & $\mathrm{Sr}^{2+}$ & $\mathrm{Ba}^{2+}$ \\
\hline $6.03(13.0)$ & $6.17(11.2)$ & $5.80(8.7)$ & $5.89(6.2)$ \\
\hline
\end{tabular}

a. $K=\left[3 \cdot \mathrm{M}^{2+}\right] /[3]\left[\mathrm{M}^{2+}\right]$.

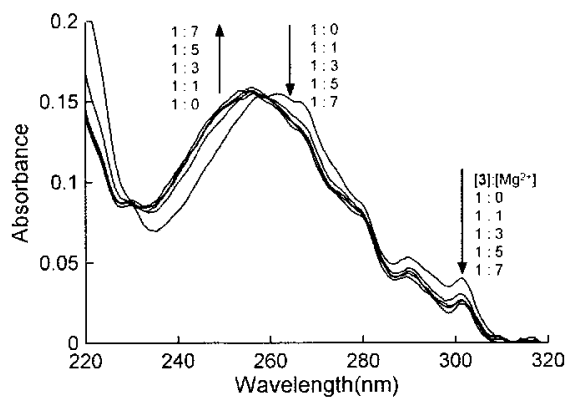

Fig. $4 \mathrm{UV}$ spectra of $\mathbf{3}$ and its $\mathrm{Mg}^{2+}$ complex in acetonitrile at $25^{\circ} \mathrm{C}$.

Figure 3 shows fluorescence spectra of compound $\mathbf{3}$ and its $\mathrm{Mg}^{2+}$ complex in acetonitrile. Fluorescence emissions from the fluorene moiety were quite weak in the absence of the $\mathrm{Mg}^{2+}$ ion. In contrast, when $\mathrm{Mg}^{2+}$ was added, 3 and $\mathrm{Mg}^{2+}$ formed a complex, and strong emission was observed. The shapes of the fluorescence spectra indicate a fluorene monomer emission, which shows the maximum intensity around $307 \mathrm{~nm}$. A similar "Off-On" behavior was also observed in the presence of other alkaline earth metal ions. The "Off-On" response capabilities were expressed as $I_{\max } / I_{0}$ values for the guest ions. They are listed in Table 1. The largest value for $\mathrm{Mg}^{2+}$ (13.0) was the strongest, and showed usefulness as a $\mathrm{Mg}^{2+}$ responsive sensor.

Figure 4 shows the UV absorption spectra of $\mathbf{3}$ and its $\mathrm{Mg}^{2+}$ complex. When $\mathrm{Mg}^{2+}$ was added, the absorbance derived from the fluorene moiety decreased slightly; its shape ( $c a .280 \mathrm{~nm}$ ) changed slightly. This result suggests that a large conformational change did not occur around the fluorene moiety. On the other hand, the absorption spectrum around 250 $\mathrm{nm}$, which was attributed to the benzo-crown moiety, showed a blue shift and increased intensity. This result can be explained by a disruption of the $\pi$-conjugated system between benzene and the amide bond at the binding event.

Figure 5 shows fluorescence excitation spectra of $\mathbf{3}$ and its $\mathrm{Mg}^{2+}$ complex. In contrast to the case of UV, the 250-nm regions from the benzene moiety almost disappeared. After the addition of $\mathrm{Mg}^{2+}$, the 268-nm wavelength response of the fluorene ring was notably increased. These results indicate that the excited-state species of $\mathbf{3}$ and $\mathbf{3} \cdot \mathrm{Mg}^{2+}$ differed from those of the ground state. They also suggest that a kind of intramolecular charge transfer occurred from the benzene moiety to the fluorene ring.

The increase of monomer emissions was dependent upon the metal-ion concentration. Complex formation constants $(K)$ were determined from the titration curves using a nonlinear least-squares curve-fitting method (Marquardt's method); ${ }^{10}$ they are listed in Table 1. These results indicate that the values of $\log K$ are not correlated well with the ionic radius. Presumably, an ionic radius of $\mathrm{Mg}^{2+}$ provided a complex structure that 


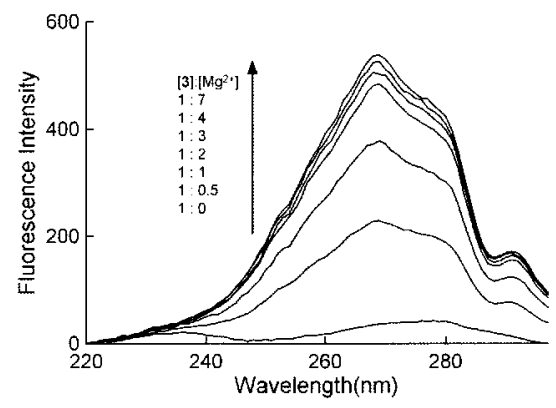

Fig. 5 Excitation spectra of $\mathbf{3}$ and its $\mathrm{Mg}^{2+}$ complex in acetonitrile at $25^{\circ} \mathrm{C}$. The respective slits for the excitation and emission monochromators were 3.0 and $5.0 \mathrm{~nm}$.

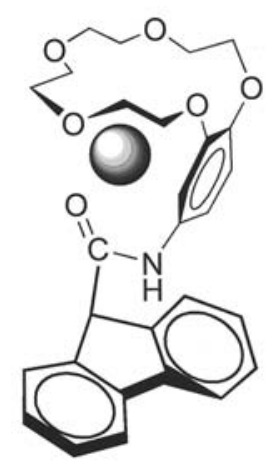

Fig. 6 Proposed complex structure of $\mathbf{3} \cdot \mathrm{Mg}^{2+}$.

showed an effective cutting of the quenching process of 3 . As above, spectral data evidently showed the structural changes of 3 upon complexation with metal ions. To clarify these changes, an ${ }^{1} \mathrm{H}$ NMR study of $\mathbf{3}$ was carried out in both the absence and presence of $\mathrm{Mg}^{2+}$ in acetonitrile- $d_{3}$ at $30^{\circ} \mathrm{C}$. Peak assignments were made by ${ }^{1} \mathrm{H}{ }^{1} \mathrm{H}$ COSY and NOESY spectra. In the $\mathbf{3} \cdot \mathrm{Mg}^{2+}$ complex (refer to the scheme for peak assignments), the crown ether moiety peaks ( $a, b, c$, and d) shifted to a lower magnetic field $(0.31,0.32,0.38$, and $0.38 \mathrm{ppm}$, respectively). The peak of amide protons (h) showed lower magnetic field shift changes $(0.38 \mathrm{ppm})$. Benzene protons ( $\mathrm{e}, \mathrm{f}$, and $\mathrm{g}$ ) shifted to a lower magnetic field $(0.25,0.20$ and $0.17 \mathrm{ppm}$, respectively). These results indicated that $\mathrm{Mg}^{2+}$ was bound cooperatively with the crown ether moiety and a carbonyl group, resulting that a molecular motion around the amide group and benzene was stopped. The chemical-shift changes of fluorene protons $(i-m)$ were not observed. This observation indicated that these protons did not receive an electron or ring-current effect upon complexation.

To examine in detail the role of carbonyl moiety at the binding event with metal ions, a ${ }^{13} \mathrm{C}$ NMR measurement was carried out in both the absence and presence of $\mathrm{Mg}^{2+}$ in acetonitrile- $d_{3}$ at $30^{\circ} \mathrm{C}$. However, the solubility of $\mathbf{3}$ was too low to measure ${ }^{13} \mathrm{C}$ NMR. Therefore, reference compound $\mathbf{4}$, which has to same binding site as $\mathbf{3}$ and sufficient solubility for acetonitrile, was employed (Fig. 1). In the $\mathbf{4} \cdot \mathrm{Mg}^{2+}$ complex, the carbonyl carbon peak shifted to a lower magnetic field (4.11 $\mathrm{ppm})$. This result clearly showed that carbonyl moiety was also complexed with $\mathrm{Mg}^{2+}$.

Based on the fluorescence and NMR studies, the expected structural change of $\mathbf{3}$ after the addition of $\mathrm{Mg}^{2+}$ on the ground state is illustrated in Fig. 6. Before complexation, the excitation of $\mathbf{3}$ was induced a charge-separation state via photo-induced electron transfer (PET) accompanied by a twisted motion between the $\mathrm{CONH}$ bond and the benzene ring. However, since the coordination of $\mathrm{Mg}^{2+}$ on the crown ether moiety and amide bond restrained the twisted motion, ICT process included it should be shut off. Thus, the fluorescence emission from fluorene can be observed. In summary, a novel fluorescent ionophore 3 based on benzo-15-crown-5 having a 9-fluorenecarboxamide moiety showed an "Off-On" fluorescent response upon the addition of $\mathrm{Mg}^{2+}$. Ionophore $\mathbf{3}$ bound $\mathrm{Mg}^{2+}$ with crown ether and the carbonyl group worked cooperatively, showing fluorescent emission. The coordination of $\mathrm{Mg}^{2+}$ in 3 induced a freezing molecular rotation around the amide bond. This $3 \cdot \mathrm{Mg}^{2+}$ structure provided effective control of the intramolecular charge-transfer process. Results suggest that $\mathbf{3}$ will be useful as a photo-detection system for $\mathrm{Mg}^{2+}$.

\section{References and Notes}

1. T. Gunnlaugsson, H. D. P. Ali, M. Glynn, P. E. Kruger, G. M. Hussey, F. M. Pfeffer, C. M. G. dos Santos, and J. Tierney, J. Fluoresc., 2005, 15, 287.

2. T. Morozumi, T. Anada, and H. Nakamura, J. Phys. Chem. B, 2001, 105, 2923.

3. T. Morozumi, H. Hiraga, and H. Nakamura, Chem. Lett., 2003, 32, 146.

4. H. Hama, T. Morozumi, and H. Nakamura, Tetrahedron Lett., 2007, 48, 1859.

5. T. Morozumi, H. Hama, and H. Nakamura, Anal. Sci., 2006, 22, 659 .

6. M. Bernius, M. Inbasekaran, E. Woo, W. Wu, and L. Wujkowski, J. Mater. Sci.: Mater. Electron., 2000, 11, 111, and references cited therein.

7. G. T. Hwang, Y. J. Seo, and B. H. Kim, J. Am. Chem. Soc., 2004, 126, 6528.

8. E. A. Englund and D. H. Appella, Org. Lett., 2005, 7, 3465.

9. 4'-(9-Fluorenecarboxamido)benzo-15-crown-5 (3): Yield $57.5 \%$. White solid. mp: $246^{\circ} \mathrm{C}$ (from $\mathrm{CH}_{3} \mathrm{COOH}$ ). ${ }^{1} \mathrm{H}$ NMR $\left(\mathrm{CD}_{3} \mathrm{CN}\right) \quad \delta=3.62(\mathrm{C}-\mathrm{CH} 2-\mathrm{O}, \mathrm{m}, 8 \mathrm{H}), 3.77$ $(-\mathrm{C}-\mathrm{CH} 2-\mathrm{O}, \quad \mathrm{m}, \quad 4 \mathrm{H}), 4.02$ (-C-CH2-O, t, 4H), 4.89 (CO-fluorene, s, 1H), 6.84 (aromatic, d, 1H), 7.00 (aromatic, d, 1H), 7.22 (aromatic, t, 1H), 7.36 (aromatic, t, 2H), 7.45 (aromatic, t, 2H), 7.64 (aromatic, d, 2H), 7.86 (aromatic, d, 2H), 8.37 (NH, s, 1H). Found: C, 70.47; H, 6.17; N, 2.90\%. Calcd. for $\mathrm{C}_{28} \mathrm{H}_{29} \mathrm{NO}_{6} \cdot 1 / 8 \mathrm{H}_{2} \mathrm{O}$ : C, 70.39; $\mathrm{H}, 6.17 ; \mathrm{N}, 2.93 \%$.

10. D. W. Marquardt, J. Soc. Ind. Appl. Math., 1963, 11, 431. 